\title{
Primera observación de murciélago bicolor (Vespertilio murinus) en la Península Ibérica al sur de los Pirineos
}

\author{
Sylvia Ortega ${ }^{1} \&$ DAVID Merino $^{1}$
}

${ }^{1}$ TESTA SL. Pl. Madrid 3-6º Izq 47001 Valladolid (Spain).

*Autor para correspondencia:_sylviaortega@testa.tv

DOI: http://dx.doi.org/10.14709/BarbJ.8.1.2015.01 (C) 2015 Published by SECEMU.

English title: First record of parti-coloured bat (Vespertilio murinus) in the Iberian Peninsula, southern the Pyrenees

recibido: $12-11-2014$ aceptado: $28-12-2014$

En la presente nota se informa del hallazgo, durante los trabajo de vigilancia ambiental en un parque eólico de la provincia de Soria, de un ejemplar hembra de murciélago bicolor (Vespertilio murinus) encontrado muerto. El hallazgo se produjo el día 26 de septiembre de 2014 en la localidad de Aguaviva de la Vega (Fig.2). Se trata de la primera hembra encontrada en la península Ibérica, lo que abre la hipótesis de un pequeño núcleo reproductor en España.

El murciélago bicolor (Vespertilio murinus) presenta una amplia distribución centroeuropea. Se distribuye desde Francia, Países Bajos, Sur de Escandinavia, Europa Central, norte y este de Siberia hasta la costa del Pacífico. El límite sur de su distribución pasa a través de la península de los Balcanes, el norte de Irán, Asia Central, Afganistán, norte de Pakistán y China. En la región mediterránea esta especie se extiende desde el sureste de Francia hacia el este por el norte de Italia y los Balcanes (Hutson et al. 2008).

La primera vez que se citó la especie en España fue en agosto de 2009 en los Pirineos, concretamente en la localidad de Sahún (Alberdi et al. 2009). Un año después, se capturaron tres machos dentro del programa de seguimiento ecológico del Parque Nacional de Ordesa y Monte Perdido, en Pirineos, uno en la localidad de Barbarruéns y los otros dos machos se capturaron en el valle de Pineta (Lorente et al. 2010). En 2011 se capturaron 13 machos de murciélago bicolor en las laderas sur del Parque Natural del Cadí. Estas citas se dan en un área poco extensa en comparación con la gran distribución mundial que tiene la especie, y en ambientes similares como son los macizos kársticos con abundantes cortados rocosos, masas forestales, y espacios abiertos al igual que ocurre con las otras citas del sureste de Europa (Pavlinic \& Tvrtkovic 2003). Todas las citas existentes en los Pirineos parecen
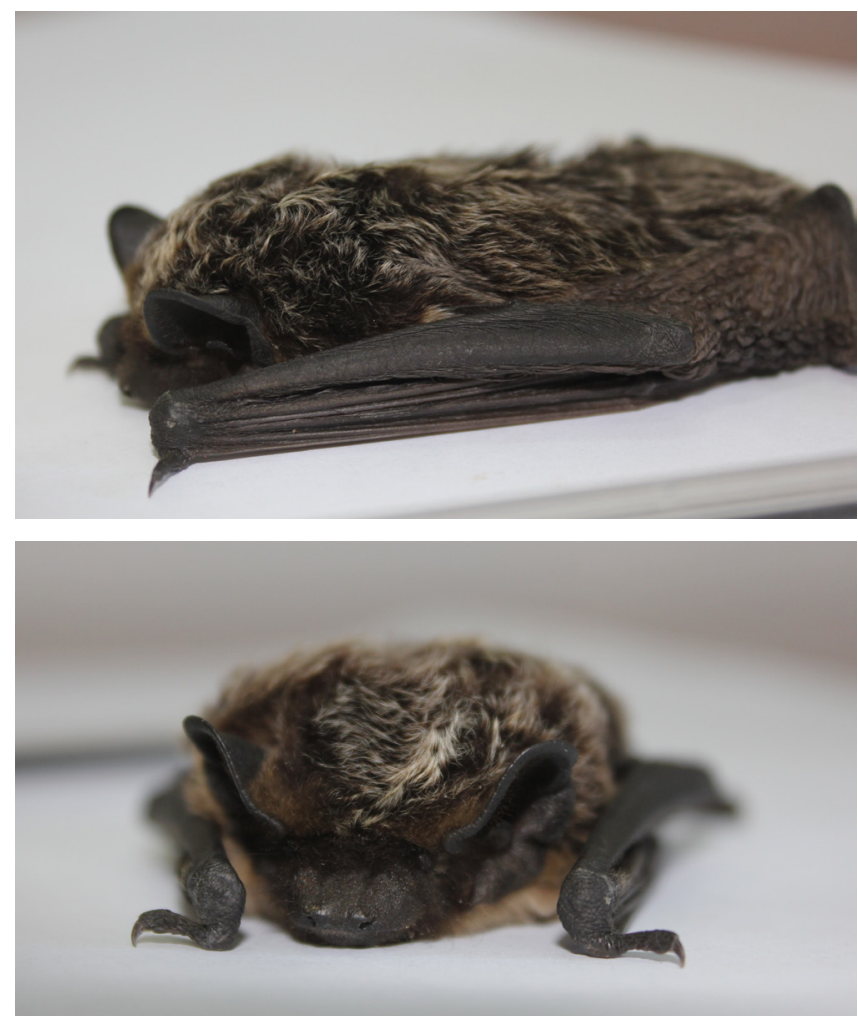

Fig. 1 - Ejemplar hallado de Vespertilio murinus

apuntar que se pueden tratar de poblaciones de machos que pasan el verano en estas zona, no siendo una especie tan escasa como se suponía (Alberdi et al. 2012).

El ejemplar hallado el 26 de septiembre de 2014 en Aguaviva de la Vega fue identificado in situ en base a la longitud del antebrazo $(45,6 \mathrm{~mm})$, la coloración del pelaje (Fig.1) y rasgos morfológicos como el trago y $\mathrm{CM}_{3}(5,8 \mathrm{~mm})$, 


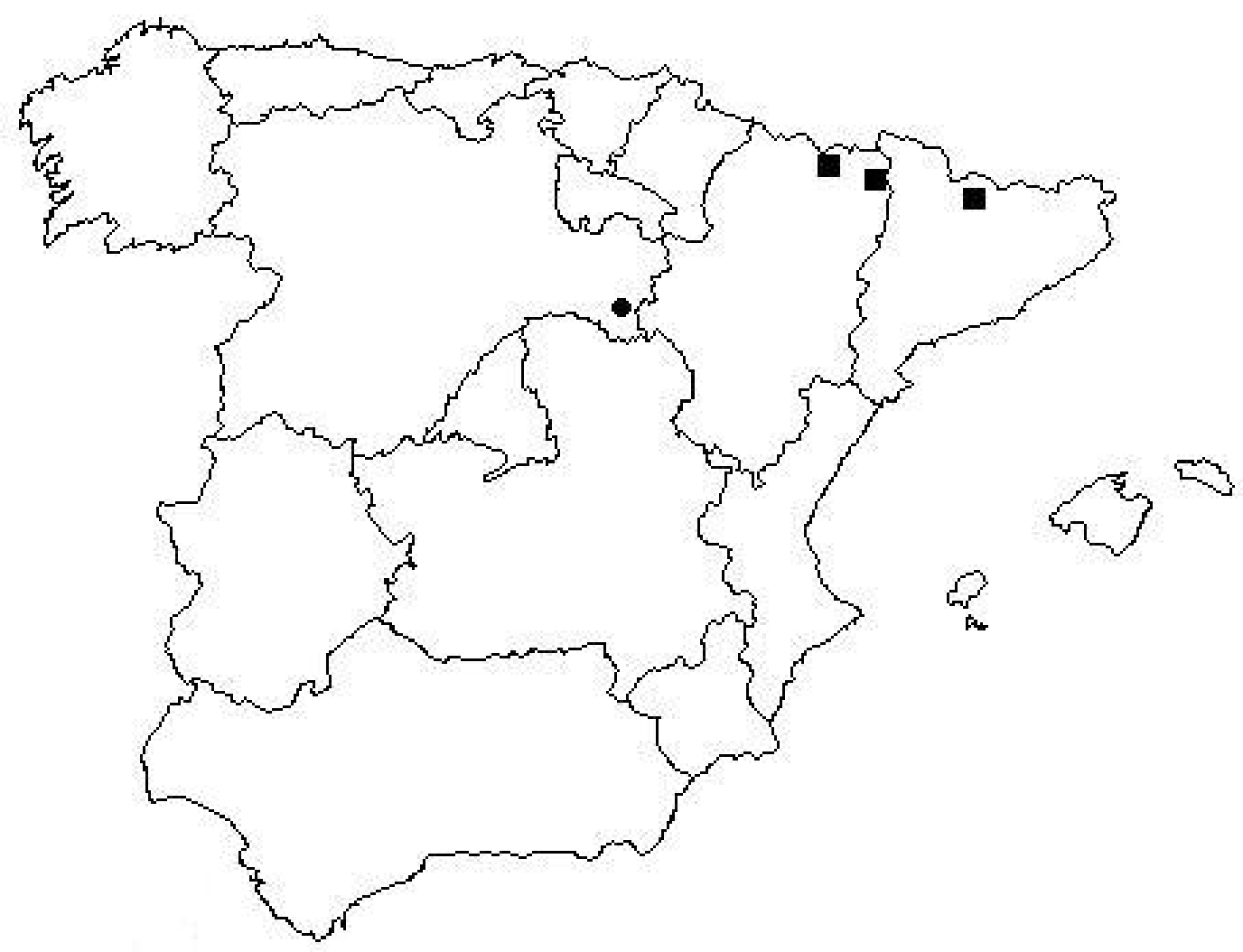

Fig. 2 - Mapa de las localizaciones de la especie en la península Ibérica. •nueva localidad • localidades ya conocidas antes de la presente nota

siguiendo las claves de Dietz et al. (2009). El ejemplar fue hallado muerto, supuestamente debido a una turbina eólica, apareciendo con relativa frecuencia ejemplares de la misma especie muertos en parques eólicos de Alemania y en menor medida en Suecia, Grecia, Francia, República Checa y Polonia (Rydell et al. 2010). Se trata de la primera hembra localizada en la península Ibérica, ya que los ejemplares capturados anteriormente en España se trataban de machos adultos. La nueva localidad se encuentra aproximadamente a $270 \mathrm{~km}$ de las otras localidades pirenaicas (Alberdi et al. 2012). El hábitat dónde ha sido encontrado el ejemplar es un encinar aclarado situado en un páramo calizo a 1189 msnm, en cuya ladera sur se encuentran pequeños roquedos y cuestas yesífereas.

Se abren varias hipótesis sobre el hallazgo del ejemplar en la meseta norte. En la primera de ellas se muestra la posibilidad de que se pueda tratar de un ejemplar en migración activa hacia algún sistema montañoso del centro peninsular, el cual bien podría ser el sistema Central. Está hipótesis se basa en la fecha del hallazgo, que se corresponde con la época de migración, y en que Vespertilio murinus es una de las cuatro especies de murciélagos europeos consideradas como migradores de largo recorrido. A fecha de hoy, se sabe que las colonias de hembras más próximas a la nueva localidad se encuentran en Neuchâtel (Suiza) (Blant \& Jaberg 1995), a unos $980 \mathrm{~km}$ al NE de Aguaviva de la Vega, encontrándose esta distancia dentro del rango de desplazamientos conocidos para la especie (hasta $1780 \mathrm{~km}$ ) (Hutterer et al. 2005). Aunque la totalidad de animales conocidos hasta ahora al SW de los Alpes son machos (Alberdi et al. 2012), un 26,6\% de las recapturas de animales anillados con largos desplazamientos de migración en toda Europa son hembras (Hutterer et al. 2005). La segunda hipótesis sobre el hallazgo del ejemplar sería la existencia de una población reproductora en los Pirineos o en el Sistema Ibérico, no descubierta aún. La última posibilidad para explicar el hallazgo sería la expansión lenta y gradual del área de distribución de la especie hacia el oeste, la cual complementa a ambas hipótesis anteriormente expuestas. Sería conveniente realizar prospecciones dirigidas a la búsqueda de esta especie, tanto en el sistema Ibérico como en el Central, al presentar hábitats adecuados para la especie.

\section{Agradecimientos}

Agradecer a la empresa TESTA SL. por facilitar la información obtenida dentro del programa de vigilancia ambiental en parques eólicos en la provincia de Soria, en especial a los compañeros Alberto Cruz y Ángel Rubio. A todas las personas que han colaborado en la confirmación de la especie: Carles Flaquer, Fabio Flechoso, Mariano García, Christian Pascual, Andrés García, Juan Tomás Alcalde, Juan Quetglas y Toni Alcocer. 


\section{REFERENCIAS}

Alberdi, A., Garin, I., Aizpurua, O. \& Aihartza, J. 2009. Primera cita confirmada del murciélago bicolor (Vespertilio murinus L., 1859) en la Península Ibérica. Resúmenes IX Jornadas de la SECEM, Bilbao, pp: 5.

Alberdi, A., Aihartza, J., Albero, JC., Aizpurua, O., López-Baucells, A., FreiXas, L., Puig-Montserrat, X., Flaquer, C. \& Garin, I. 2012. First records of the parti-coloured bat Vespertilio murinus (Chiroptera: Vespertilionidae) in the Pyrenees. Mammalia 76 (2012): 109-111. Doi: http://dx.doi.org/10.1515/ mamm.2011.106

Blant, J.D., \& JaberG, C. 1995. Confirmation of the reproduction of Vespertilio murinus L. Switzerland. Myotis, 32-33: 203-208

Dietz, C., Von Helversen, O. \& Nill, D. 2009. Bats of Britain, Europe and Northwest Africa. A\&C Black, London. pp. 400.

HutTereR, R. 2005. Bat migrations in Europe: a review of banding data and literature. German Agency for Nature Conservation, Bonn, Germany.

Hutson, A.M., Spitzenberger, F., Aulagnier, S., Coroiu, I., Stubbe, M., Ariunbold, J., Buuveibaatar, V., Dorjderem, S., Monkhzul, T., Otgonbaatar, M. \& Tsogbadrakh, M. 2008. Vespertilio murinus. The IUCN Red List of Threatened Species. Version 2014.3.

Lorente, L., Albero, J.C., Rivas, J.L. \& Jato, R. 2010. Nuevas observaciones de Murciélago bicolor ( Vespertilio murinus L., 1758) en el Pirineo aragonés. Galemys 22: 53 - 54 .

Pavlinic, I. \& TVRTKovic, N. 2003. The presence of Eptesicus nilssonii and Vespertilio murinus in the Croatian bat fauna confirmed. Natura Croatica, 12(2): 55-62.

Rydell, J., Bach, L., Dubourg-Savage, M. J., Green, M., Rodrigues, L., \& Hedenström, A. 2010. Bat mortality at wind turbines in northwestern Europe. Acta Chiropterologica, 12(2): 261-274). Doi: http:// dx.doi.org/10.3161/150811010X537846 